\title{
The Genetic Diversity of Eight Chicken Populations Assessed by 102 Indels Markers
}

\author{
Aye A. Maw ${ }^{1}$, Takeshi Shimogiri ${ }^{2}$, Kai Yamamoto $^{2}$, Kotaro Kawabe ${ }^{3}$, \\ Koichiro Hamada $^{4}$, Yasuhiro Kawamoto ${ }^{5}$ and Shin Okamoto ${ }^{2}$ \\ ${ }^{1}$ United Graduate School of Agricultural Science, Kagoshima University, Kagoshima 890-0065, Japan \\ ${ }^{2}$ Faculty of Agriculture, Kagoshima University, Kagoshima 890-0065, Japan \\ ${ }^{3}$ Frontier Science Research Centre, Kagoshima University, Kagoshima 890-0065, Japan \\ ${ }^{4}$ Kagoshima Prefectural Institute for Agricultural Development (KIAD), Kagoshima, 899-4461, Japan \\ ${ }^{5}$ Faculty of Agriculture, University of the Ryukyus, Okinawa, 903-0213, Japan
}

This is the first study in which genetic diversity of eight chicken populations including Satsumadori, Satsumajidori and three populations each of improved and commercial chicken by using 102 indels markers. Both of Satsumadori and Satsuma-jidori were referred to as Satsuma's chickens in this study. The proportion of polymorphic loci, average observed and expected heterozygosities were ranged from 0.500 to 0.814 , from 0.147 to 0.257 and from 0.175 to 0.247 , respectively. Monomorphic indels loci being specific to a particular population were detected in some populations. The coefficients of genetic differentiation (Gst) of the Satsuma's chickens and six populations of improved and commercial chickens were 0.124 and 0.287 , respectively. The Gst for all populations was 0.329 . The $(D s)$ genetic distance matrixes for all possible pairs (28 pairs) of populations were estimated from allele frequency to construct UPGMA tree. The UPGMA tree clearly divided Satsuma's chickens and the other populations. The 2-D scatter plots of PCA analysis assembled the individuals into their respective populations.

Key words: commercial chicken, genetic diversity, improved chicken, indels markers, Satsumadori, Satsuma-jidori

\section{Introduction}

Satsumadori is a well-known Japanese indigenous breed native to Satsuma region. Satsuma used to be the former name of the Kagoshima Prefecture in Southern Kyushu. Satsumadori was a breed of game chicken developed by crossing with Shamo and Shokoku (Catalogue of Asian native chicken, 1991). As the appearance of Satsumadori was beautiful with abundant long tail feather, the breed was designated a natural monument in 1943 (Catalogue of Asian native chicken, 1991). In the year 1990, the Kagoshima Prefectural Institute for Agricultural Development (KIAD) began to develop a new variety of the local Satsuma chickens by inter-breeding between the male Satsumadori and the female Rhode Island Red. In the year 2000, the project was completed and resulted in the Satsuma-jidori. This new variety distinguished for its fine muscle fiber, a light crunchy texture, good meat color and lean meat. Since they are more

Received: June 18, 2012, Accepted: October 15, 2012

Released Online Advance Publication: November 25, 2012

Correspondence: Prof. Dr. S. Okamoto, Faculty of Agriculture, Kagoshima University, Korimoto 1-21-24, Kagoshima 890-0065, Kagoshima, Japan.

(E-mail : chacha@agri.kagoshima-u.ac.jp) resistant to disease and heat compared to ordinary broilers, Satsuma-jidori chicken has set a new benchmark for the industry. Today Satsuma-jidori meat is well-known brand in Japan. Due to these excellent genetic characteristics of Satsumadori and Satsuma-jidori, it is special interest to assess the genetic variation between these two chicken populations from Satsuma region and other chicken populations by utilizing modern molecular tools.

The genetic variability and relationship of Japanese and foreign chicken has been assessed by microsatellite DNA profiling (Osman et al., 2006). The genetic relationship between the Japanese native chicken (Satsumadori and Ingie) and the commercial chickens has been evaluated using 70 chicken autosomal SNP genotypes by the DigiTag 2 assay (Shimogiri et al., 2011).

The insertion and/or deletion (indels) polymorphisms are diallelic markers with a potential characteristics for use in the study of genetic diversity and phylogenic relationship. Indels markers have many genetic advantages for analytical use: they are widely spread throughout the genome, all of the polymorphisms derive from a single mutation event and they have reduced mutation rates (Natalle et al., 2010). In our previous study, we analyzed the genetic diversity of Myan- 
mar and Indonesia native chickens together with Red Junglefowl (Gallus gallus bankiva) and Green Junglefowl (Gallus varius) by using 102 indels polymorphisms (Maw et al., 2012). We found that the genetic variability is higher among native chicken populations and lower in Red Junglefowl and Green Junglefowl. The native chickens from two countries were genetically close to each other and remote from Red Junglefowl and Green Junglefowl from Java Island.

In this study, we emphasized on 102 indels polymorphisms from previous study as genetic markers to assess the genetic diversity and to determine the genetic relationship of two chicken populations from Satsuma region and other chicken populations.

\section{Materials and Methods}

\section{Chicken Samples}

In total, 474 birds of two chicken populations from Satsuma region and six chicken populations representing three improved and three commercial chicken populations were examined. Two chicken populations from Satsuma region were Satsumadori $(\mathrm{SD}, n=20)$ and Satsuma-jidori (SJ, $n=40)$. Three improved chicken populations were Rhode Island Red (RIR, $n=55$ ), White Leghorn (WL, $n=$ $60)$ and Barred Plymouth Rock (BPR, $n=119$ ). Three commercial chicken populations were Ross (RS, $n=60)$, Cobb $(\mathrm{CB}, n=60)$ and Boris Brown (BB, $n=60)$.

Satsumadori and Satsuma-jidori (both of them were referred to as Satsuma's chickens) samples were obtained from Kagoshima Prefectural Institute for Agricultural Development (KIAD). Genomic DNA was extracted from blood samples using standard phenol- chloroform extraction protocols (Sambrook et al., 1989).

\section{Genotyping of Indels Markers}

Information and genotyping method of the 102 indels markers used in this study ( $\mathrm{m} 1$ to $\mathrm{m} 102$ ) have been reported in our previous study (Maw et al., 2012). Genotypes of indels polymorphisms were determined by size difference between the PCR fragments. As for genotypes, $A$ and $B$ denoted insertion and deletion alleles respectively.

\section{Statistical Analysis}

The genetic variability of each population was assessed by calculating the minor allele frequency (MAF), proportion of polymorphic loci ( $P_{\text {poly: }}$ Lewontin and Hubby, 1966), and average observed and expected heterozygosity $\left(H_{E}\right.$ : Nei, 1978). Chi square $\left(\chi^{2}\right)$ approximation was used to test HardyWeinberg Equilibrium (HWE) (Weir, 1996) and calculate the degree of genetic differentiation (Gst) (Nei, 1973). The pair wise standard genetic distance $(D s)$ (Nei, 1972) was computed using PHYLIP ver. 3.69 (Felsenstein, 2009). Then we constructed phylogenetic tree by using unweighted pair group method with arithmetic mean (UPGMA) (Sneath and Sokal, 1973) implemented by MEGA software ver. 4.1 (Tamura et al., 2007). To examine population substructure, the individual genotype for 102 indels were subjected to a principal component analysis (PCA) using EIGENSOFT version 3.0 (Patterson et al., 2006).

\section{Results}

\section{Indels Polymorphisms}

Out of the 102 indels markers, 98 were polymorphic in the 474 chickens and the remaining four were monomorphic. Of the 98 loci, 36 loci were fixed in SJ whereas 47 loci were fixed in SD excluding four monomorphic markers for all populations. Then 35 loci in RIR, 37 loci in WL, 41 loci in BPR, 20 loci in RS, 16 loci in $\mathrm{CB}$ and 24 loci in $\mathrm{BB}$ were fixed. We observed one $(\mathrm{m} 31 ; B B=1)$ was monomorphic only in the SJ whereas six $(\mathrm{m} 6 ; B B=1, \mathrm{~m} 9 ; A A=1, \mathrm{~m} 22 ; A A$ $=1, \mathrm{~m} 45 ; B B=1, \mathrm{~m} 47 ; A A=1, \mathrm{~m} 94 ; B B=1)$ only in the SD. In addition, two was monomrphic only in RIR (m10; $B B=$ $1, \mathrm{~m} 91 ; A A=1)$, five only in WL $(\mathrm{m} 5 ; A A=1, \mathrm{~m} 16 ; A A=$ $1, \mathrm{~m} 71 ; B B=1, \mathrm{~m} 77 ; A A=1, \mathrm{~m} 92 ; B B=1)$, and in BPR $(\mathrm{m} 4 ; B B=1, \mathrm{~m} 25 ; B B=1, \mathrm{~m} 46 ; A A=1, \mathrm{~m} 50 ; B B=1, \mathrm{~m} 82$; $B B=1)$. Then two was monomorphic only in RS (m1; $B B=$ 1, $\mathrm{m} 14 ; A A=1)$.

In this study, $80 \%$ of the polymorphic loci had a minor allele frequency (MAF) of $>10 \%$ and $49 \%$ of $>20 \%$. The average MAF by 98 polymorphic markers was estimated as 0.227. Tests of Hardy-Weinberg equilibrium (HWE) over all experimental populations and within populations indicated that all of the populations were in HWE except SJ at m6.

\section{Genetic Variability}

The genetic variability of eight chicken populations was demonstrated in Table 1. The $P_{\text {poly }}$ value of each population was ranged from 0.500 to 0.814 (Table 1). The RS, CB and $\mathrm{BB}$ populations showed higher values than other populations. The SJ population showed higher value $(0.608 \pm 0.048)$ than SD $(0.500 \pm 0.049)$ but lower than RIR $(0.618 \pm 0.048)$.

The $\bar{H}_{\mathrm{O}}$ and $\bar{H}_{\mathrm{E}}$ values were ranged from 0.147 in SD to 0.257 in $\mathrm{BB}$ and from 0.175 in SD to 0.247 in RS. The RS, $\mathrm{CB}$ and $\mathrm{BB}$ populations showed higher values than other populations. The SJ population showed higher $\bar{H}_{\mathrm{O}}$ and $\bar{H}_{\mathrm{E}}$ values $(0.196$ and 0.212$)$ than SD $(0.147$ and 0.175$)$ and RIR (0.153 and 0.177$)$. The $\bar{H}_{\mathrm{E}}$ values of $\mathrm{CB}, \mathrm{WL}$ and RIR ( 0 . $245,0.192$ and 0.177 , respectively) were consistent to our previous study using 98 autosomal SNP markers (Riztyan et al., 2012).

The degree of genetic differentiation (Gst) value was calculated as 0.124 in Satsuma's chickens and 0.287 among the other chicken populations. The Gst value for over all populations was 0.329 .

\section{Phylogenetic Analysis}

The UPGMA tree constructed from the $D s$ distances matrixes between eight chicken populations calculated from 102 indels markers in Fig. 1. Satsuma's chicken populations were genetically related with each other with the smallest $D s$ value of 0.072 . The UPGMA tree divides 8 chicken populations to two major clades. The first clade was composed of the improved and commercial chicken populations and the second clade was composed of Satsuma's chicken populations.

\section{Population Substructure by using PCA Analysis}

The 2-D scatter plot of the first two principal components 
Table 1. Genetic variability of eight chicken populations

\begin{tabular}{|c|c|c|c|c|c|c|c|}
\hline Populations & $n$ & $P_{\text {poly }} \pm \mathrm{SE}$ & $\bar{H}_{\mathrm{O}} \pm \mathrm{SE}$ & $\bar{H}_{\mathrm{E}} \pm \mathrm{SE}$ & $\bar{H}_{\mathrm{T}}$ & $\bar{H}_{\mathrm{S}}$ & $G_{s t}$ \\
\hline Satsuma-jidori (SJ) & 40 & $0.608 \pm 0.048$ & $0.196 \pm 0.004$ & $0.212 \pm 0.004$ & & & \\
\hline Satsumadori (SD) & 20 & $0.500 \pm 0.049$ & $0.147 \pm 0.004$ & $0.175 \pm 0.004$ & & & \\
\hline Rhode Island Red (RIR) & 55 & $0.618 \pm 0.048$ & $0.153 \pm 0.004$ & $0.177 \pm 0.004$ & & & \\
\hline White Leghorn (WL) & 60 & $0.598 \pm 0.048$ & $0.203 \pm 0.005$ & $0.192 \pm 0.004$ & & & \\
\hline Barred Plymouth Rock (BPR) & 119 & $0.549 \pm 0.049$ & $0.160 \pm 0.004$ & $0.176 \pm 0.004$ & & & \\
\hline Ross (RS) & 60 & $0.765 \pm 0.042$ & $0.243 \pm 0.005$ & $0.247 \pm 0.004$ & & & \\
\hline Cobb (CB) & 60 & $0.814 \pm 0.038$ & $0.237 \pm 0.005$ & $0.245 \pm 0.004$ & & & \\
\hline Boris Brown (BB) & 60 & $0.716 \pm 0.045$ & $0.257 \pm 0.005$ & $0.225 \pm 0.004$ & & & \\
\hline Satsuma's chickens & 60 & $0.686 \pm 0.046$ & & & 0.220 & 0.193 & 0.124 \\
\hline Improved and commercial chickens & 414 & $0.941 \pm 0.023$ & & & 0.295 & 0.211 & 0.287 \\
\hline Total Population & 474 & $0.961 \pm 0.042$ & & & 0.307 & 0.206 & 0.329 \\
\hline
\end{tabular}

Satsuma's chickens: SJ and SD.

Improved chickens: RIR, WL and BPR.

Commercial chickens: RS, CB and BB.

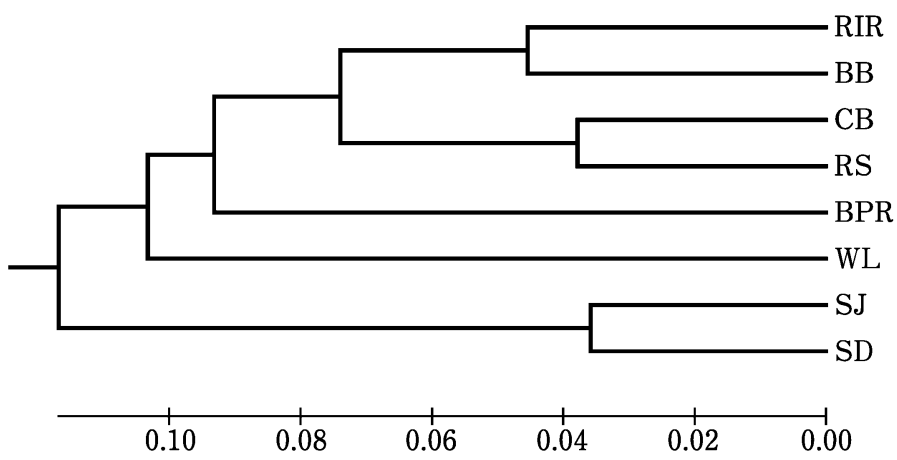

Fig. 1. UPGMA tree constructed by genetic distance matrices from 8 chicken populations by using 102 indels markers. SJ=Satsumajidori, $\mathrm{SD}=$ Satsumadori, RIR $=$ Rhode Island Red, $\mathrm{WL}=$ White Leghorn, $\mathrm{BPR}=$ Barred Plymouth Rock, $\mathrm{RS}=$ Ross, $\mathrm{CB}=$ Cobb, $\mathrm{BB}=$ Boris Brown.

(PCs) was showed in Fig. 2. Contribution ratios of PC1 and PC2 were 13.8 and $10.7 \%$, respectively. By considering PC1 and PC2 cumulatively, individuals were grouped, corresponding to their respective populations. In addition, Satsuma's chicken populations showed admixture between them whereas RIR did not show admixture to SJ. The two commercial chickens (RS and CB) were closely positioned near each other, which was consistent to UPGMA tree in Fig. 1.

\section{Discussion}

The present study examined the genetic diversity of eight chicken populations by using 102 indels polymorphisms as genetic markers. Out of 102 indels loci, 98 were polymorphic over the whole populations. This was almost the same number as our previous study (Maw et al., 2012). The MAF percentage in present study was close to the previous study of Väli et al. (2008); using indels as genetic markers in wolf populations showed $74 \%$ of the polymorphic loci had
MAF of $>10 \%$ and $49 \%$ of $>20 \%$.

The genetic variability based on $P_{\text {poly }}$ and heterozygosities was higher in commercial (RS, $\mathrm{CB}$ and $\mathrm{BB}$ ) and $\mathrm{SJ}$ than improved (RIR, WL and BPR) and SD chickens populations. These results support hybridization of breeds when establishing commercial chickens and SJ by interbreeding between $\mathrm{SD}$ and RIR. In addition, the layers (WL and BB) showed lesser genetic variability than the broilers (CB and RS). These results were consistent with those obtained by microsatellites and SNPs in earlier studies (Crooijmans et al., 1996; Vanhala et al.,1998; Hillel et al., 2003; Shimogiri et al., 2012).

For genetic relationships, the UPGMA dendrogram showed that the Satsuma's chickens and the group of chickens representing improved and commercial chicken populations were genetically distant with each other (Fig. 1). The highest Gst value (0.329) supported their genetic relationship. The Gst value (0.289) was also high among the improved and commercial chicken populations. These re- 


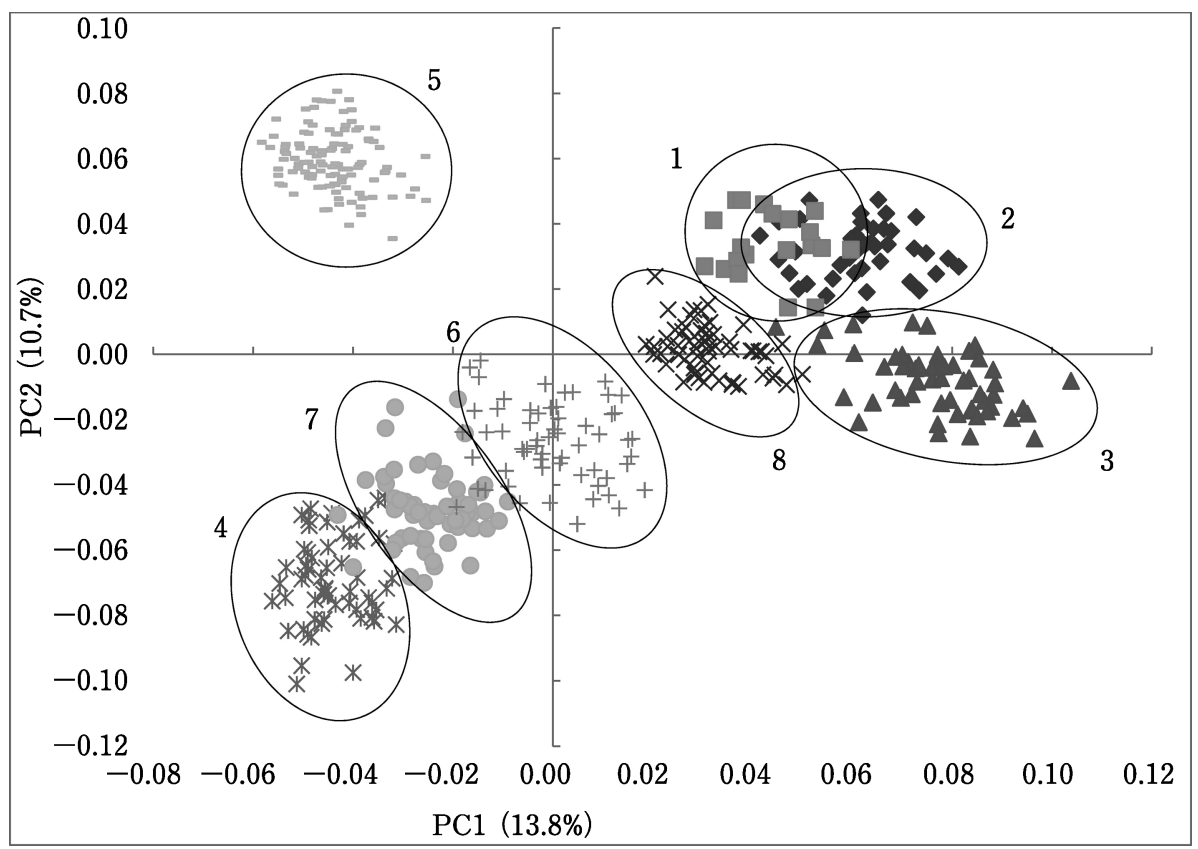

Fig. 2. 2-D scatter plot of the first two principal components (PC1 and PC 2) for 474 individuals of eight chicken populations based on 102 indels genotypes. $1=$ Satsuma-jidori, $2=$ Satsumadori, $3=$ Rhode Island Red, $4=$ White Leghorn, $5=$ Barred Plymouth Rock, $6=$ Ross, 7 $=$ Cobb, $8=$ Boris Brown.

sults might be the result of intensive selection to fix desirable traits during the process of improvement in commercial chickens. Satsuma's chickens were shown as closely related to each other in the dendrogram. However, RIR, one of the parental breeds of SJ, was genetically distant from the Satsuma's chickens in the dendrogram (Fig. 1). The similar results were obtained from the 2-D plot of PCA analysis (Fig. 2) where individuals from RIR population were not showed admixture to SJ. These may be resulted from differences in sample collection. The SD samples were taken from a parental stock of SJ in KIAD while RIR were not.

In the Japanese chicken market, some brand chickens are directly associated with pure breeds or particular hybrids. Therefore, DNA test for breed or brand identification is realistic and desirable for types of brand chicken. In the report of Takahashi et al. (2007) five microsatellite markers which have fixed allele in Nagoya chicken were used to discriminate between Nagoya breed and all other chicken. Rikimaru and Takahashi (2007) reported the use of 14 microsatellite markers, which were fixed in Hinai-jidori to distinguish Hinai-jidori chicken (Hinai-dori X Rhode Island Red) from other chicken breeds. Nakamura et al. (2010) stated that the utilization of one fixed fragment size in five microsatellite markers to check the authenticity of Nagoya breed-labeled chicken on the market. Currently, there is no application on indels markers for breed identification. In this study, 36 of the 102 markers were fixed in the SJ chickens, some of which were not fixed in other chickens. This finding may provide a possibility for distinguishing the SJ from others.

In summary, the results reported here were in good reflection to the characteristics of populations and agreement to previous reports. Therefore, indels polymorphisms were convenient and useful markers to assess the genetic diversity and genetic relationships of chicken populations.

\section{Acknowledgments}

The authors gratefully thank to Associate Professor Yamashita Hideji (Tokai University, Faculty of Agriculture).

\section{References}

Catalogue of the Native Poultry of Southeast Asia. FFTC Book Series No. 43. 1991.

Crooijmans RP, Groen AF, Van Kampen AJ, Van der Beek S, Van der Poel JJ and Groenen MA. Mirosatellite polymorphism in commercial broiler and layer lines estimated using pooled blood samples. Poultry Science, 75: 904-909. 1996.

Felsenstein J. Phylogeny Inference Package Version 3.69. 2009.

Hillel J, Groenen MA, Tixier-Boichard M, Korol AB, David L, Kirzhner VM, Burke T, Barre-Dirie A, Crooijmans RP, Elo K, Feldman MW, Freidlin PJ, Maki-Tanila A, Oortwijin M, Zthomson P, Vignal A, Wimmers $\mathrm{K}$ and Weigend S. Biodiversity of 52 chicken populations assessed by microsatellite typing of DNA pools. Genetics Selection Evoluation, 35: 533-557. 2003.

Lewontin RC and Hubby JL. A molecular approach to the study of genetic heterozygosity in natural populations of Drosophila 
pseduobscura. Genetics, 54: 595-609. 1966.

Maw AA, Shimogiri T, Riztyan, Kawabe K, Kawamoto Y and Okamoto S. Genetic diversity of Myanmar and Indonesia native chickens together with two Jungle Fowl species by using 102 Indels polymorphisms. Asian-Australasian Journal of Animal Science, 25: 927-934. 2012.

Nakamura A, Nagao K, Watanabe H and Kondo H. Characteristics of PCR fragments amplified using five microsatellite markers for identifying the Nagoya breed. Animal Science, 81: 438443. 2010.

Nei M. Genetic distance between populations. American Naturalist, 106: 283-292. 1972.

Nei M. Analysis of gene diversity in subdivided populations. Proceedings of the National Academy of the Sciences of the United States of America, 70: 3321-3323. 1973.

Nei M. Estimation of average heterozygosity and genetic distance from a small number of individuals. Genetics, 89: 583-590. 1978.

Natalle SCF, Resque RL, Ribeiro-Rodrigues EM, Guerreiro JF, Santos NPC, Santo AR and Santos S. X-linked insertion/deletion polymorphisms: Forensic applications of a 33-markers panel. International Journal of Legal Medicine, 124: 589-593. 2010.

Osman SAM, Sekino M,Nishihata A, Kobayashi Y, Takenaka W, Kinoshita K, Kuwayama T, Nishibori M, Yamamoto Y and Tsudzuki M. The Genetic Variability and Relationships of Japanese and Foreign Chickens Assessed by Microsatellite DNA Profiling. Asian-Australasian Journal of Animal Sciences, 19: 1369-1378. 2006.

Patterson N, Price AL and Reich D. Population structure and eigenanalysis. Public Library of Science- Genetics, 2: 20742093. 2006.
Riztyan, Nishida T, Worawut R, Shimogiri T, Maw A A, Kawabe K, Kawamoto Y and Okamoto S. Genetic variation and phylogeographic analysis of native chicken populations in Myanmar and Thailand. Journal of Poultry Science, 49: 68-73. 2012.

Rikimaru $\mathrm{K}$ and Takahashi H. A method for discriminating a Japanese brand of chicken, the Hinai-dori, using microsatellite markers. Poultry Science, 86: 1881-1886. 2007.

Sambrook J, Fritsch EF and Maniatis T. Molecular cloning: A laboratory manual, $2^{\text {nd }}$ edition, Cold Spring Laboratory Press, Cold Spring Harbor, New York, USA, 1989.

Shimogiri T, Nishida N, Kudo M, Niwa K, Nishibori M, Kinoshita K, Okamoto S, Maeda Y, Tokunaga K and Yasue H. Genetic relationship between Japanese native and commercial breeds using 70 chicken autosomal SNP genotypes by the DigiTag2 assay. Animal Genetics, 43: 98-103. 2011.

Sneath PHA and Sokal RR. Numerical Taxonomy. Freeman WH: San Francisco, 1973.

Takahashi $\mathrm{H}$ and Nakamura A. Genetic diversity and differentiation of the Nagoya breed inferred from microsatellite DNA polymorphisms. Journal of Poultry Science, 44: 135-140. 2007.

Tamura K, Dudley J, Nei M and Kumar S. MEGA4: Molecular evolutionary genetics analysis (MEGA) software version 4.0. Molecular Biology and Evolution, 24: 1596-1599. 2007.

Väli U, Brandström M, Johansson M and Ellegren H. Insertiondeletion polymorphisms (indels) as genetic markers in natural populations. BMC Genetics, 9: 1-8. 2008.

Vanhala T, Tuiskula-Haavisto M, Elo K, Vilkki J and Maki-Tanila A. Evaluation of genetic variability and genetic distances between eight chicken lines using microsatellite markers. Poultry Science, 77: 783-790. 1998.

Weir BS. Genetic Data Analysis II. Sinauer. Sunder land, MA, 1996. 